\title{
Acromegaly incidentally diagnosed at term in a pregnant woman presenting with ventricular premature complexes
}

\author{
Aiswarya K. Nair*, Haritha Sagili, Jayalakshmi Dorairaj, T. Parvathi
}

Department of Obstetrics and Gynecology, JIPMER, Puducherry, India

Received: 24 May 2021

Accepted: 16 June 2021

*Correspondence:

Dr. Aiswarya K. Nair,

E-mail: ashgokulam@gmail.com

Copyright: ( $)$ the author(s), publisher and licensee Medip Academy. This is an open-access article distributed under the terms of the Creative Commons Attribution Non-Commercial License, which permits unrestricted non-commercial use, distribution, and reproduction in any medium, provided the original work is properly cited.

\section{ABSTRACT}

Pituitary adenomas lead to increased growth hormone production and acromegaly. Pregnancy in acromegaly is rare as spontaneous conception is affected. We presented a case of 31 year old lady conceived by ovulation induction and presented at term gestation with ventricular premature complexes and was subsequently diagnosed with pituitary micro adenoma causing acromegaly. There has been no reported case of pregnancy with acromegaly presenting with premature ventricular complexes. Moreover, diagnosis of acromegaly in pregnancy is difficult and limited literature is available on its effects as well as management.

Keywords: Acromegaly, Gestational hypertension, Pituitary neoplasms, Pregnancy, Ventricular premature complexes

\section{INTRODUCTION}

Acromegaly has an annual incidence between 0.2 and 1.1 cases/100,000 population. ${ }^{1}$ Pregnancy in women with acromegaly is rare and the literature is limited to case reports/few case series. We described a case of acromegaly incidentally diagnosed at term in a pregnant woman presenting with ventricular premature complexes, which has not been reported previously.

\section{CASE REPORT}

A 31 year old primigravida presented with breathlessness and palpitations at 37 weeks of gestation in the year 2020 . There was no history of fever, chest pain, hemoptysis, syncope or nocturnal cough. She was diagnosed with gestational hypertension at 32 weeks and blood pressure was controlled with labetalol $100 \mathrm{mg}$ bd. She had conceived with second cycle of ovulation induction after 10 years of primary infertility. Her antenatal period was uneventful. On examination, coarse facial features, prognathism, large tongue, unusually thick/full lips, enlarged hands and feet were noted (Figure 1).
There was no pallor, BP was $130 / 70 \mathrm{mmHg}$ and per abdomen uterus was term and fetal heart rate was good. Holter ECG showed ventricular premature complexes and echocardiography showed mildly dilated left atrium/ventricle, mild mitral/aortic regurgitation, with normal left ventricular systolic function (Figure 2). Chest $\mathrm{X}$-ray was normal and COVID testing was negative.

Blood investigations were normal except for hypokalemia upto $2.75 \mathrm{meq} / \mathrm{l}$, potassium supplementation was given after which ventricular premature complexes subsided. Based on appearance, hypertension, hypokalemia and history of infertility, a diagnosis of pituitary adenoma causing acromegaly was suspected. An endocrinology opinion was sought. 24 hour urine potassium excretion was $12 \mathrm{meq} /$ day (normal range 25-30 meq/day). Oral glucose tolerance test, visual field and fundus examination were normal. Her thyroid profile was normal with borderline fT4 levels. The blood cortisol level was normal. Non-contrast MRI brain revealed a faint hypo enhancing lesion $\left(7 \times 8 \mathrm{~mm}^{2}\right)$ in the right anterior pituitary suggestive of pituitary micro adenoma (Figure 3). Conservative management was planned. 

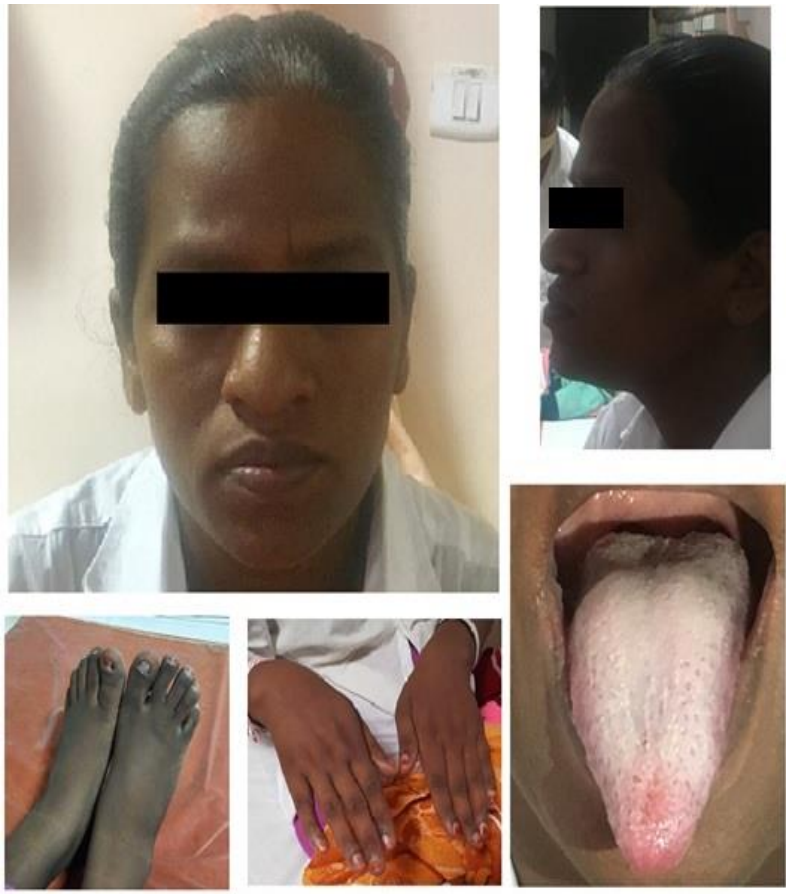

Figure 1: Acromegalic features like facial features, prognathism, large tongue, unusually thick/full lips, enlarged hands and feet.

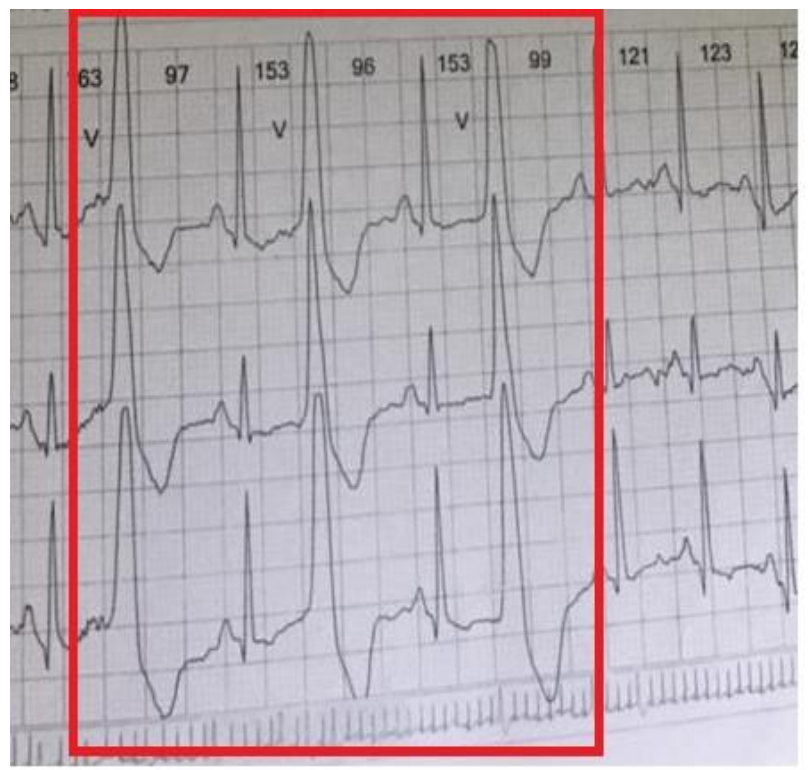

Figure 2: Holter ECG with ventricular premature complexes.

An elective caesarean was performed at 38 weeks in view of precious pregnancy under combined spinal-epidural anesthesia and a healthy male baby weighing $3.75 \mathrm{~kg}$ was delivered. There were no perioperative complications. Postnatally, her blood pressures were stabilized and labetalol was discontinued by day 2 . In view of nonfunctioning micro adenoma, she was planned for endocrinology evaluation at 3 months postpartum and was discharged on the tenth postnatal day.

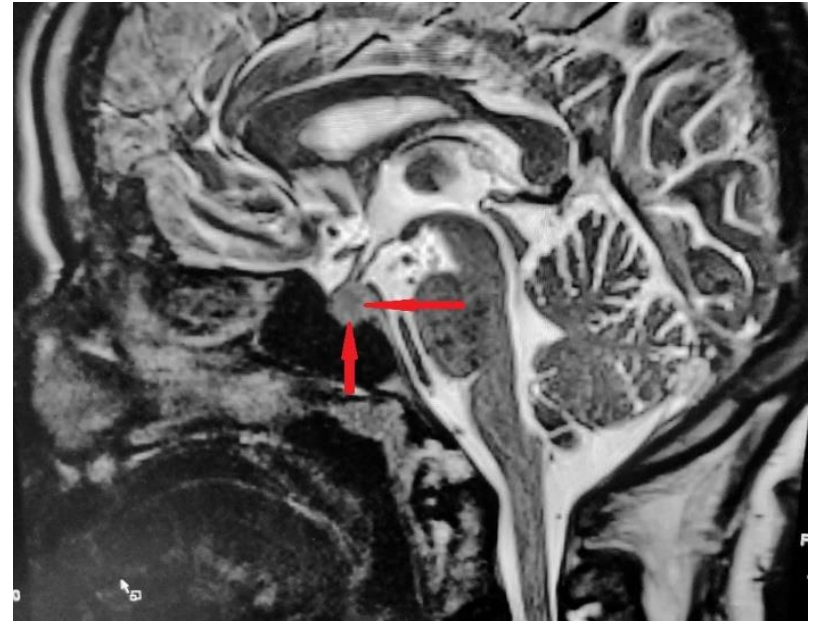

Figure 3: Non-contrast MRI brain showing a faint hypo enhancing lesion $\left(7 \times 8 \mathrm{~mm}^{2}\right)$ in the right anterior pituitary suggestive of pituitary micro adenoma.

\section{DISCUSSION}

Acromegaly is associated with infertility due to hyperprolactinemia, gonadotrophin deficiency and compressive effects of the tumour. ${ }^{2}$ The incidence of gestational diabetes and hypertension is increased in pregnant women with acromegaly due to the effects of excess growth hormone on insulin resistance and sodium retention. ${ }^{3}$ Hypertrophic cardiomyopathy is present in about one third of these patients. ${ }^{4}$ However, there has been no report of pregnant acromegalic patient presenting with ventricular premature complexes previously.

Pregnancy alters tumor size and hormone secretion, complicating the evaluation and management of patients with pituitary neoplasms. The influence of treatment modalities on the developing fetus also affects therapeutic decision making. ${ }^{5}$

Hormonal assessment of women with acromegaly during pregnancy is unreliable due to interference of placental and pituitary hormones. The usual reference ranges of $\mathrm{GH}$ and IGF-1 levels for non-pregnant women cannot be extrapolated to pregnancy. Hence, the definite evaluation of acromegaly should be postponed until puerperium due to the lack of normative data for both GH and IGF- 1 levels during gestation. ${ }^{4}$

Management of pregnancy with acromegaly is expectant and the withdrawal of medical therapy is recommended with close follow up. Monthly follow up is recommended for all patients, with visual field testing in every trimester for macro adenomas. After delivery, MRI must be repeated to evaluate the tumor size. ${ }^{6}$

Our patient presented at term for the same, hence conservative management was planned. Our dilemmas were the mode of delivery and the type of anesthesia. Considering the intracranial tumor, precious pregnancy 
and baby weight, caesarean was planned. As there was no evidence of raised intracranial pressure, combined spinalepidural anesthesia was considered.

Based on a review by Chanson et al in 2019, pregnancy appears to be uneventful, except for increased incidence of gestational hypertension and diabetes. When acromegaly is incidentally diagnosed during pregnancy close monitoring and imaging are necessary as the tumor may increase in size and cause symptoms. In the rare cases of tumor enlargement during pregnancy, medical treatment with dopamine agonists or somatostatin analogs may be tried before resorting to transsphenoidal surgery. ${ }^{4}$

\section{CONCLUSION}

Pregnancy with acromegaly is rare and management is challenging in the scenario of pituitary adenoma. A multidisciplinary approach is necessary to provide optimal care for this high-risk pregnancy.

Funding: No funding sources Conflict of interest: None declared Ethical approval: Not required

\section{REFERENCES}

1. Lavrentaki A, Paluzzi A, Wass JA, Karavitaki N. Epidemiology of acromegaly: review of population studies. Pituitary. 2017;20(1):4-9.

2. Hannon AM, O'Shea T, Thompson CA, Hannon MJ, Dineen R, Khattak A, et al. Pregnancy in Acromegaly is safe and is associated with improvements in IGF-1 concentrations. Eur J Endocrinol. 2019;180(4):21-9.

3. Abucham J, Bronstein MD, Dias ML. Management of endocrine disease: acromegaly and pregnancy: a contemporary review. Eur J Endocrinol. 2017;177(1):1-12.

4. Chanson P, Vialon M, Caron P. An update on clinical care for pregnant women with acromegaly. Expert Rev Endocrinol Metab. 2019;14(2):85-96.

5. Huang $\mathrm{W}$, Molitch ME. Pituitary tumors in pregnancy. Endocrinol Metab Clin North Am. 2019;48(3):569-81.

6. Araujo PB, Neto LV, Gadelha MR. Pituitary tumor management in pregnancy. Endocrinol Metab Clin North Am. 2015;44(1):181-97.

Cite this article as: Nair AK, Sagili H, Dorairaj J, Parvathi T. Acromegaly incidentally diagnosed at term in a pregnant woman presenting with ventricular premature complexes. Int J Reprod Contracept Obstet Gynecol 2021;10:2907-9. 